\title{
Extraits de La Neige sur l'herbe de Youri Norstein
}

Introduction : Eugénie Zvonkine

Traduction des extraits : Anne-Marie Passaret

Copyright images et texte russe : Youri Norstein

\section{Introduction}

La Neige sur l'berbe [Sneg na trave] de Youri Norstein sort en 2008, édité par la fondation Youri Norstein et l'éditeur Krasnaïa plochtchad avec le soutien de la Banque nationale russe (Sberbank Rossii), dont Norstein écrit en plaisantant qu'on l'appelle dans son équipe « le ministère de la Culture ${ }^{1} »$. Il s'agit de deux volumes imposants, agrémentés de nombreux dessins, croquis, reproductions ainsi que d'un index d'illustrations qui donne en un coup d'œil l'amplitude des inspirations et références qui travaillent le cinéma de Norstein : des fresques de Lascaux jusqu'à René Magritte et Pablo Picasso en passant par Hokusai et la peinture populaire russe de Gorodets.

Le livre est dédié par Norstein à son épouse et sa plus proche collaboratrice, Frantcheska Iarbousova. L'introduction au premier volume nous indique clairement la visée de l'ouvrage :

Il y a une vieille histoire drôle, qui a un rapport direct avec le cinéma d'animation. Le patient d'un hôpital psychiatrique écrit quelque chose et son voisin lui demande :

1.Jurij Norštejn, 2008 ; Sneg na trave, Fond Jurija Norštejna, Krasnaja Ploščad', Moscou, p. 5 . 
SLOVO

254 À l'Est de Pixar : le film d'animation russe et soviétique - no 48/49

«Que fais-tu?

- J'écris une lettre, ne me dérange pas.

- Une lettre à qui ?

— J'écris à moi-même. Laisse-moi tranquille.

- Et qu'est-ce que tu t'écris à toi-même ?

- Je ne sais pas, je n'ai pas encore reçu la lettre. »

Faire un film, c'est s'écrire à soi-même une lettre que l'on recevra après le carton $\ll$ Fin $\gg^{2}$.

Cette introduction métaphorique, qui pourrait fonctionner pour toute œuvre d'art en train de se faire, suggère ainsi que c'est ce processus de création que Norstein nous invite à découvrir au gré des pages.

Ce n'est donc pas tant les films achevés que le cinéaste analyse, même si l'introduction à l'ouvrage propose une réflexion plus théorique (chapitre « Le phénomène de la représentation $\gg$ ), que le processus de réflexions, de choix et d'efforts consécutifs qui permettent aux films de naître tels que nous les connaissons. De ce point de vue, l'inachèvement participe pleinement du principe même de la composition de cet ouvrage, Norstein consacrant la majeure partie du deuxième volume à son film inachevé Le Manteau [Šinel'] d'après Nicolas Gogol.

Norstein se penche sur ses sources d'inspiration. Par exemple, c'est un chat peint sur une chaise auquel on prend les yeux pour le petit lapin de La Renarde et le Lièvre [Lisa i zajac] (1973). C'est une rencontre muette du cinéaste lui-même avec sa belle-mère avec qui il est fâché qui inspire les chorégraphies des silhouettes du héron et de la cigogne, renfrognés dans le brouillard automnal du Héron et la Cigogne [Žuravl' i caplja] (1974). La maison du Conte des contes [Skazka skazok] (1979) « vient » de la maison d'enfance de Norstein lui-même.

Norstein montre aussi la manière dont le film se prépare, qu'il s'agisse du scénario ou du rythme du film à venir : pour Le Petit Hérisson dans le brouillard [Ëžik v tumane] (1975), nous avons ainsi de véritables partitions rythmiques qui permettent de comprendre comment le cinéaste et son équipe anticipent les vitesses d'animation.

Norstein explique également comment se fabriquent et se conçoivent ses dispositifs d'animation : l'animation à plusieurs planches qui donne la sensation de la profondeur de champ aux images du Petit Hérisson dans le brouillard ou à toute la rue reconstruite pour Le Manteau qui permet de voir évoluer le personnage dans des décors picturaux et complexes.

2.Ibid., p. 3 . 
Entrer dans les détails habituellement secrets de la fabrication des films donne de nouveaux outils pour travailler et comprendre les œuvres elles-mêmes : dans le volume 2 , nous voyons ainsi à la fois la taille des petits éléments qui composent la silhouette d'Akaki Akakievitch et la minutie avec laquelle ils sont fabriqués, ce qui permet de comprendre comment le cinéaste anticipe le «jeu d'acteur» de son personnage.

Les extraits traduits ici ne donnent évidemment pas une vision exhaustive du processus de création de Youri Norstein et de son équipe mais proposent une première excursion dans les coulisses de la fabrication de ses films.

\section{EXTRAITS}

\section{«Si l'on prenait les lèvres de Nikanor Ivanovitch pour les coller sous le nez d'Ivan Kouzmitch... » (I, p. 12)}

À quel moment commence la représentation à l'écran, c'est un mystère. Où est le premier point à partir duquel on peut dire : c'est ici que commence un film d'animation. Je ne le sais pas. Je sais seulement qu'il y a une feuille blanche, tandis que dans le cinéma en prise de vues réelles, il n'y en a pas, la caméra tombe toujours sur une table, une maison qui sont déjà là... Que l'on prenne un acteur, qu'on le grime à outrance jusqu'à le métamorphoser, on prend de la matière vivante. Et nous, que prenons-nous ? Rien de rien. Qu'est-ce que cela veut dire ? Que l'art de l'animation est monstrueux parce qu'il faut tout créer, depuis la première ligne sur un espace blanc jusqu'à la dernière bande-son lors de l'enregistrement. Tout est à créer. Rien ne nous est donné à l'avance. C'est terrible, mais exaltant. La différence entre le film d'animation et le film en prises de vue réelles est décrite dans La Noce de Gogol, dans le monologue d'Agafia Tikhonovna : «Si on prenait les lèvres de Nikanor Ivanovitch pour les coller sous le nez d'Ivan Kouzmitch, si on amalgamait le tout avec les manières dégagées de Balthazar Balthazarovitch et qu'on ajoute encore la corpulence d'Ivan Pavlovitch... » C'est typiquement un procédé du cinéma d'animation. Aucun autre art ne peut en faire usage, nous sommes les seuls à pouvoir le faire. L'écrivain aussi, bien sûr, car il octroie à son héros divers traits qu'il emprunte un peu à chacun. Heinrich Heine en a très bien parlé. Il aimait déambuler sur les boulevards et observer les passants. Il cultivait son boulevard comme un jardin botanique, regardait les dames dont les rondeurs débordaient du corset et à la vue de leur embonpoint « il était pris de mal de mer ». Mais cela ne l'empêchait pas d'utiliser ce parc zoologique pour reconstituer des fragments en un tout. Toujours chez Gogol, dans La Confession d'un auteur et à propos du Revizor: « [...] je décidai de faire un ramassis de tout ce qu'il y avait, à ma connaissance, de mauvais en Russie, de toutes les injustices qui se commettent là où la justice est de 
SLOVO

256 À l'Est de Pixar : le film d'animation russe et soviétique - no 48/49

rigueur, et de tourner en ridicule tout à la fois. » C'est ce que le film d'animation a toute liberté de réaliser : un ramassis.

L'animation pour moi n'est pas du tout du cinéma. Ce qui la relie au cinéma, c'est seulement le procédé de transposition d'une image sur une pellicule et le déroulement de cette pellicule dans un projecteur-des procédés chimiques et optiques. Pour le reste, c'est un art bizarre, que je peux comparer au théâtre, et surtout à la littérature, malgré la nature totalement représentative du film d'animation. Pourquoi le théâtre ? Dans son rapport à une convention et au surnaturel, par exemple. Nous savons bien que le théâtre n'est qu'une boîte sombre, la scène est un trou noir, et qu'il suffit d'un son de la nature, le sifflet d'un train ou la sirène d'un bateau pour que cette obscurité de la scène prenne vie. La force du film d'animation ressemble à cela. C'est à travers une convention que l'on perçoit la réalité et qu'on a la joie immédiate de la reconnaître. Ceci est impossible dans le cinéma en prises de vues réelles, dont la mission est autre. Tandis qu'au théâtre, un geste vrai sur fond d'une convention fait déferler sur nous la lave de la réalité. Nous nous prêtons consciemment à cette expérience. Le fait que notre conscience soit prête à se transporter dans un autre espace montre à l'évidence pour quel art incroyable et stupéfiant nous travaillons.

Et bien sûr, « Une belle fable m'arrachera des larmes ${ }^{3}$. »

Plus il y a d'imprévu et d'invention, mieux cela vaut pour le film d'animation, qui fixe sur une pellicule des secrets de la conscience et du sentiment. Aucun intermédiaire ne fait obstacle à la représentation pour refléter nos sentiments, si on ne tient pas compte de ce qui est lié à la réalisation concrète du film. La représentation est une fonction de l'action. Les sentiments, reflets d'une matière naturelle qu'ils transposent en métaphores, sont une condition obligatoire de n'importe quelle création et la condition essentielle du film d'animation.

Je voudrais organiser ces rencontres (I, p. 19)

Je voudrais organiser ces rencontres de telle sorte qu'on parle du cinéma d'animation comme faisant partie de la culture générale, des beaux-arts et du cinéma mondiaux. Nous parlerons du concept du temps comme de l'une des catégories de l'art du cinéma et du film d'animation en particulier. Nous évoquerons le concept du temps dans les arts statiques - la peinture et la sculpture. Et naturellement, nous en viendrons au film d'animation, à la fabrication d'un film; pour savoir quels éléments (personnage, espace, lumière, son, parole, texture, pause, jeu, etc.)

3. Vers du poème de Pouchkine Élégie (n.d.t.). 
participent à la construction de l'ensemble et se fondent dans l'énergie commune du film. De quelle façon le tout se forme à partir d'un échange d'énergies des différents éléments, comment sa représentation influence le jeu d'un personnage, comment un personnage se construit en fonction de son jeu, comment la représentation elle-même peut devenir la dramaturgie d'un film. Quelles sont les spécificités du film d'animation, quand s'y révèle un mystère que nous avons du mal à nous expliquer complètement. Mais nous pourrons découvrir des conditions qui nous permettent de faire du cinéma, et nous évoquerons la base théorique du film d'animation.

\section{Mä̈akovski matérialise le mot (I, p. 16)}

Le mot est pour lui un objectif de caméra qui transforme la réalité. J'en ai été le témoin. Un jour, je me trouvais dans une énorme cantine d'ouvriers. La cantine était une immense salle, 300 ou 400 mètres carrés, bondée, noire de monde. Je me sentis vite mal, comme si quelqu'un me comprimait par en haut avec un poids lourd. Je ne comprenais pas ce qui se passait, car tout le monde était assis à table sans bouger, or je sentais quelque chose peser très fort sur mes épaules. J'appris par la suite que c'était le jour de la paie, les hommes discutaient ferme, leur chope de bière ou leur verre de vodka à la main. Malgré leur position statique, le ton montait. En outre, l'entrée de la salle était fantastique. Au milieu du vestibule, émergeait d'une montagne de chapeaux et de manteaux une gigantesque sculpture en plâtre

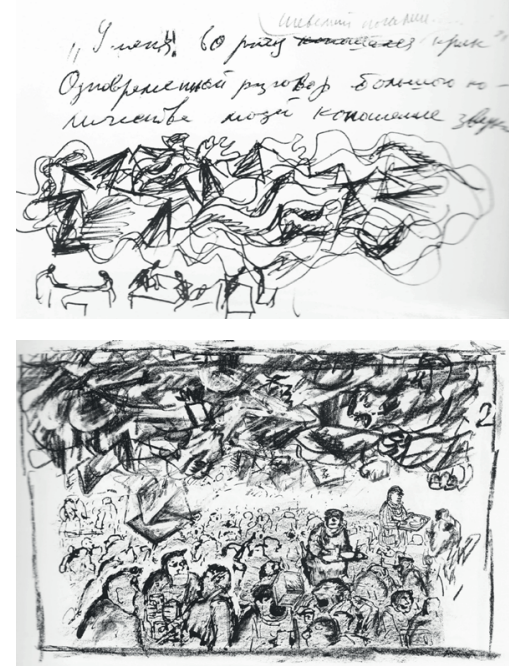

Figures 1 et 2 de Lénine, repeinte des dizaines de fois pour des fêtes officielles. Comme à l'ordinaire, des odeurs de borchtch, de nouilles, de boulettes de viande, de sirop, émanaient des gamelles distribuées. Et dans cette cantine, où des hommes étaient assis pratiquement sans bouger, le grondement de leurs voix graves se matérialisait sous le plafond, sous forme d'une grosse nuée, et s'exprimait plastiquement.

Un dessin de cette situation pourrait ressembler à cela : une masse condensée en hauteur, dont les composants se heurtent, et qui cliquète comme des cubes que l'on déplace.

Une énorme nuée de petits cubes au-dessus de la tête des hommes qui parlent. Parfois, au milieu de la nuée, apparaîssent ici ou là une bouche, une oreille, ou une main renforçant le son. La masse sonore tournoie, et ses composants s'entrechoquent. 
SLOVO

258 À l'Est de Pixar : le film d'animation russe et soviétique - nº 48/49

Figure 3

Des cubes sonores s'élèvent lentement de la bouche de ceux qui parlent, puis ils retombent lentement sur leurs têtes

(fig. 2 et 3 ).

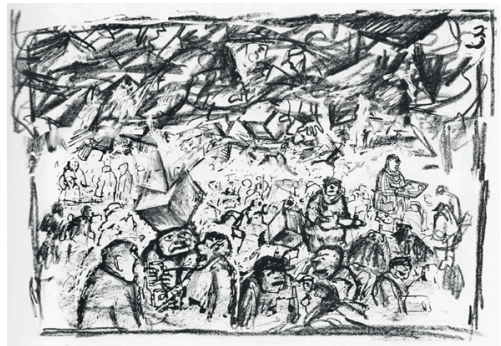

Ils tombent doucement, sans faire trop mal, mais on les sent suffisamment pour recevoir le coup de l'argument qui a été lancé dans la conversation et pour y répondre en cognant le voisin (fig. 4 et 5). Là, j'ai vu Maïakovski. Une masse cubo-futuriste s'amoncelle au plafond et bouge lentement et les arêtes des cubes s'entrechoquent, elle tressaille, se fragmente. Le son a acquis des traits visibles.

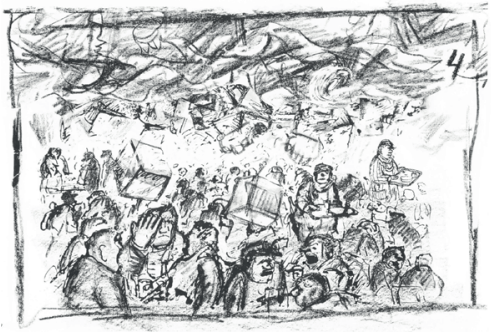

Figure 4

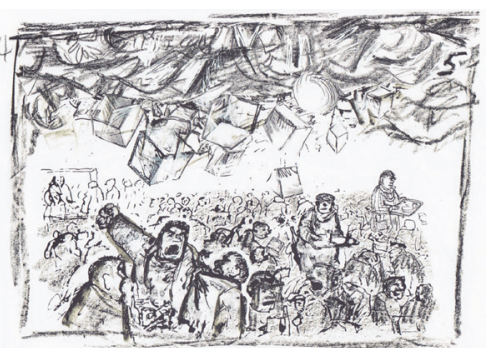

Figure 5

\section{Hokusai (I, p. 44-45)}

Nous prendrons pour exemple une estampe d'Hokusai, dont la légende est «Contemplation de la lune ». Le plan fixe de cette composition peut durer une, deux ou trois minutes si l'on trouve un soutien sonore. Je vous pose la question : que choisiriez-vous comme bande-son pour pouvoir regarder cette composition comme un séquence de film ?

\section{- Peut-être un bruitage?}

- Quel genre de bruit?

- Peut-être du Debussy? 
- Debussy? Non, c'est trop beau. L'homme admire la lune, qu'ajouterait Debussy? Vous devez chercher un contrepoint. On peut évidemment introduire un bruit de cigales, mais ce n'est pas suffisant. D'une façon générale, il est bon que le sublime s'abaisse à un niveau plus prosaïque, il en devient alors encore plus sublime. Alors, réfléchissez encore, quelle bande son pour cette composition ? Un homme est assis, il a abandonné toutes ses activités domestiques et regarde la lune. Quel son pourrait bien soutenir cela ?

- Le bruit des vagues?

- Oui, c'est bien, mais pas parfait. Il y a des vagues sur la gravure, et moi je parle de contrepoint. Cela est présent dans la poésie japonaise...

\section{- Les échos lointains d'une fête?}

- Une fête ? C'est déjà mieux, nous donnons de l'espace au plan. Mais regardez plus attentivement l'air content de l'homme. Il a tout laissé pour s'asseoir là et admirer la lune; sa femme, ses enfants sont rentrés et sa femme hurle : «Ko-seï-san! Où est-il, cet idiot ?» Alors, nous avons une sensation beaucoup plus aiguë de sa sérénité et de l'espace vivant. Aussitôt nous comprenons qu'il n'a pas fini son travail, que les enfants n'ont pas eu à manger, que le feu n'est pas ranimé... Et lui, voyez-vous, il admire la lune, quel idiot, il a bien besoin de regarder la lune, cet idiot, alors qu'on a tellement besoin de lui à la maison. Le ton n'est pas méchant, parce que c'est une scène qui se répète chaque jour.

Lorsque nous passons un plan fixe, en réalité ce n'est pas de l'immobilité, le plan continue de se dérouler, mais il est nécessaire de trouver des éléments sonores qui vont lui permettre de durer davantage.

Frantcheska (I, p. 109-110)

Un jour, je regardais des gravures japonaises.

Frantcheska m'a fait vivre un événement stupéfiant en me montrant comment un papillon naît de la chrysalide. Elle avait apporté à la maison un bouquet d'orties, qui craquait à cause des mâchoires de dizaines de chenilles qui mangeaient les feuilles. Ensuite, elles se dispersèrent dans toute la pièce et s'accrochèrent où elles pouvaient, et très vite, au lieu de chenilles, il y avait des chrysalides. Frantcheska me dit : «Un papillon va sortir de la chrysalide ». Les membranes de la chrysalide 
SLOVO

260 À l'Est de Pixar : le film d'animation russe et soviétique - no 48/49

s'ouvraient et se refermaient, j'en frémissais d'émotion. C'était un spectacle cosmique. J'étais témoin de l'apparition d'une nouvelle vie. Les membranes de la chrysalide s'ouvrirent de nouveau et s'immobilisèrent. Il y eut un silence. Des ailes mouillées émergèrent de la carapace, fripées comme le corps d'un bébé qui vient de naitre. Puis, de la chrysalide sortit le nouveau-né. La seconde phase du spectacle consistait à remplir cet être de temps. Pour un être humain, cela prend deux ou trois ans, dans le cas présent, cela a pris une heure et demie. Le papillon s'est mis à gonfler d'air liquéfié ses minces vaisseaux et les nervures de ses ailes, formant des longerons. Cette liqueur s'est durcie, les ailes se sont redressées et se sont tendues comme des voiles au vent. Elles étaient d'une grande pureté, aucune brindille de fleur n'y était mêlée, aucune poussière ne s'était encore posée sur ce petit être tout neuf. Les ailes, élastiques, se sont mises à battre en souplesse, puis à trembler. Comme un avion qui vérifie ses moteurs et son levier de vitesse, avant de décoller. « Le papillon se prépare à voler, il sèche ses ailes. »-commentait Frantcheska. Elle anticipait chaque mouvement du papillon car elle en avait élevé des quantités dans l'enfance, sur des orties, sur des branchages de chêne. Je pense qu'elle-même a été autrefois papillon, et chardon, et laurier de Saint-Antoine... Comme l'héroïne de Gabriel Marquez autour de laquelle volètent des papillons.

Frantcheska respire le monde en croissance des plantes, des fleurs, des êtres vivants, elle le connaît comme pouvait le connaître un peintre de la Renaissance.

Je me souviens de mon étonnement lorsque pour Le Conte des contes je lui dis : «Frania ${ }^{4}$,j'ai besoin d'un arbuste mouillé avec un enfant emmailloté dessous ». Au bout de dix minutes, elle ressortit avec une esquisse d'arbuste mouillé, en s'essuyant les mains.

Il n'y a là aucun dédoublement, aucune fracture du moi intérieur, mais tout simplement l'idéal.

Quand je parle de Frantcheska, je cite toujours les vers de Shakespeare dans Le Marchand de Venise : « Cette nuit-là, Médée marchait dans les champs, à la recherche d'herbes magiques afin de rendre la jeunesse au vieux Jason... » à un observateur extérieur, nos conversations devaient paraitre énigmatiques et incompréhensibles. « Ici, il me faut une herbe empoisonnée et là, de l'absinthe au milieu d'un tas de métal rouillé, ou encore un petit clou de girofle... une palissade à l'abandon aux boules d'or ternies... ». Je doute qu' il existe à Soïouzmoultfilm un autre dessinateur connaissant aussi bien qu'elle la nature. Elle prépare un plan comme une liqueur faite maison. Elle sait quand le dessin doit être gratté, quand il faut amollir la matière, quand il faut étaler avec le coude. Ici, il faut de l'hyper-réalisme et là, une goutte de

4. Diminutif de Frantcheska (n.d.t.). 
pluie sur une branche fine, il faut qu'il fasse nuit et que ça sente le bleu-nuit, et que la nuit se dissolve. Il faut qu'au pied du perron il y ait des fleurs à l'odeur de nuit. Frantcheska dessine en toute liberté parce qu'elle connaît ces fleurs-là. Elle réalise une image comme on prépare une potion. Un autre dessinateur pourrait croquer de magnifiques fleurs, avec une grande précision, avec tous les détails, mais il y manquerait l'odeur.

\section{La Renarde et le Lièvre (I, p. 97)}

Adapter simplement un conte populaire à l'écran est une entreprise vouée à l'échec, alors on doit garder l'idée d'un drame psychologique. Le matériau du conte s'est forgé à travers les siècles, on ne peut rien lui ajouter, on ne peut rien lui retrancher, on ne peut que le suivre à la lettre. On n'obtiendra rien sans un travail intérieur, sans un recoupement avec son propre état d'âme. En faisant ce conte, en me soumettant aux règles du conte populaire, je pensais pourtant à ce pauvre Lièvre, à son sentiment d'avoir été offensé. L'histoire du Lièvre était mon histoire.

Venons-en au personnage. En tournant attentivement les pages du livre sur la peinture de Gorodets, mon attention fut retenue par l'image du chat.

Un chat imposant, plein de dignité, qui ne nous quitte pas de ses gros yeux, assis sur la chaise où il est dessiné. Il serait impossible de s'asseoir sur cette chaise sans offenser cet animal. Je montrai le chat à Franceska : «Voilà les yeux de notre Lièvre ». Ne pensez surtout pas que nous avons transplanté mécaniquement une peinture du folklore dans notre film. À la vue de ce chat, j'ai immédiatement imaginé la ligne générale du film et compris que le regard de ces yeux invraisemblables pouvait servir de trame d'un bout à l'autre du film. Le Lièvre pouvait scruter la salle depuis l'écran comme on le voit souvent sur les dessins d'enfant.

Un montage s'inspirant de la peinture sur des rouets anciens et le regard du lièvre sortant de l'écran : je tenais la cohérence du film (fig. 6).

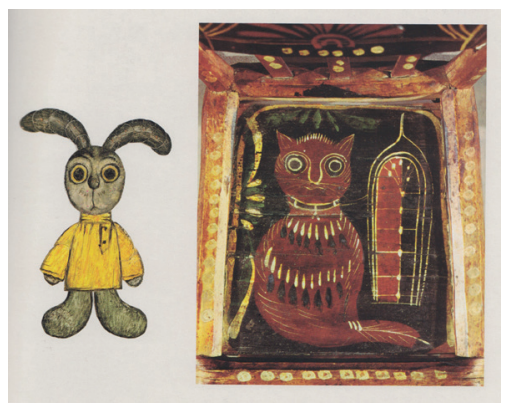

Figure 6

Le lapin dessiné par Franceska, 1973, et le siège d'une chaise peinte par I. A. Mazine, 1920 
SLOVO

262 À l'Est de Pixar : le film d'animation russe et soviétique - no 48/49

\section{Le Héron et la Cigogne (I, p. 127)}

La naissance d'un film reste un mystère inexplicable. La sensation physiologique du plan apparaît non pas quand il prend forme dans les recoins de ma conscience, mais quand l'image se « fait entendre ».

Ce cinéma, imaginé et rêvé au fond de ma conscience, je le connais depuis mon film Le Héron et la Cigogne. Je dois dire que je n'éprouve jamais de joie particulière avant de commencer un nouveau film. Au contraire, je sens que je me condamne à endurer de nouvelles souffrances. Je suis partagé entre la peur et le désir de faire ce film. Ma Frantcheska cite alors le dicton : «Les yeux s'affolent, mais les mains travaillent. »

Le sujet du film n'a rien de compliqué, il est proche, me semble-t-il, de la poésie de Tchékhov et de Gogol. C'est à dessein que j'emploie le mot « poésie ». C'est une

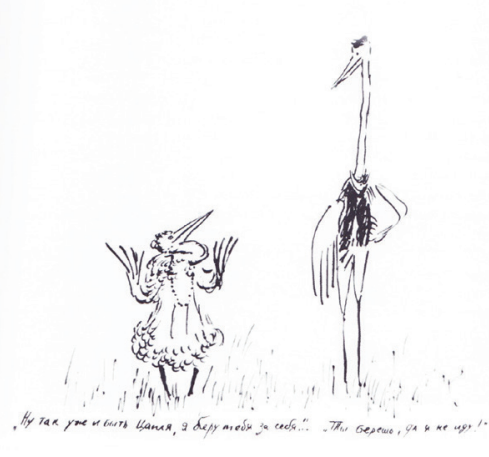

Figure 7 histoire, somme toute, ordinaire - deux êtres ne peuvent ni construire ni asseoir leur bonheur parce que chacun, craignant d'être humilié par l'autre, essaie de montrer son indépendance et sa supériorité. L'histoire est plus humaine qu'animale. C'est là qu'ont surgi les problèmes que je pressentais mais dont j'ignorais qu'ils seraient si compliqués. J'avais lu ce conte dans mon enfance, puis l'avais lu à mes enfants. Mais j'ai eu envie d'en faire un film lorsque le bruissement de l'herbe et la rumeur des roseaux au bord de la rivière se sont imposés à moi. C'est étrange, car notre art est visuel, mais c'est un son qui a été le déclencheur du film. Avant l'écriture du scénario, je me suis représenté la scène suivante : le héron et la cigogne se chipotent, l'un dit « $\mathrm{Ha}$ » et l'autre reprend « $\mathrm{Ha} »$ (fig. 7 ).

\section{Le héron et la cigogne}

Je ne savais pas encore où serait placée cette scène, mais je la dessinai. Vous vous souvenez de mon interrogation sur la naissance d'un film : d'où vient-il ? Quelle en est l'origine? Le Héron et la Cigogne donne une réponse éloquente. Précisons qu'il faut tout de même écrire un scénario. Mais il est bon que le réalisateur soit co-auteur. Que se passerait-il s’il prenait connaissance du scénario sans connaître le vécu de l'auteur? 
Je le répète encore, le désir de raconter doit provenir de sa propre expérience de vie. C'est l'expérience personnelle qui va fournir les détails du film. Une expérience de vie, pas une expérience artistique. La vie ne se vit qu'une fois, l'expérience de vie est unique, elle ne s'ancre pas dans une habitude. L'expérience artistique a tendance à créer des habitudes, c'est-à-dire à introduire une certaine apathie dans le travail. Le métier, qui est une qualité indispensable, abolit l'immédiateté de la découverte.

J'ai ensuite écrit moi-même une sorte de scénario, même si ça n'y ressemblait pas tout à fait. J'ai plutôt noté des moments de l'action, rien de plus, tout en étant sans cesse accablé par un doute: comment ne pas tomber dans le zoomorphisme, comment faire pour que cette histoire colle à la nature humaine. Par la suite, c'est Roman Katchanov qui a écrit le scénario, j'en ai extrait quelques détails et j'ai fait le film d'après mes croquis. Dans le générique, nous apparaissons comme les deux scénaristes. Roman Katchanov est un merveilleux réalisateur. Ses films La Moufle, Le Crocodile Guena, Tchebourachka, La Vieille Chapoclac, sont des chefs-d'œuvre de l'animation. Il a été l'un de mes maîtres. C'est quelqu'un d'une grande bonté, qui a beaucoup de tact, et qui maîtrise parfaitement la technique du jeu dans le cinéma d'acteurs. Beaucoup de nos réalisateurs d'animation sont passés « par ses mains ». Je ne pense pas me tromper en disant que Roman Katchanov a créé une école du mouvement dans l'animation avec marionnettes. C'est grâce à lui que je suis devenu réalisateur.

\section{Le problème du décor (I, p. 132-135)}

Il faut créer dans le plan des conditions aussi simples que possible pour qu'une certaine action y prenne place et pas une autre. En y réfléchissant, on peut même se dire qu'on doit faire le film dans le rectangle blanc de l'écran. Un simple rectangle blanc, c'est aussi un espace. Il est important d'en venir à ce résultat. Sous le terme « espace », je n'entends pas seulement un paysage spécifique. Là, nous avons eu de gros problèmes avec Le Héron et la Cigogne.

Car ce sont des oiseaux des marais, alors, quelle habitation leur donner ? Si on choisit un nid dans le marais, on tombe dans la zoologie. Si on leur fabrique une maison, cela est stupide, car la cigogne a un long cou, il faudrait que cela soit une maison très haute qui ressemblerait plutôt à des WC au milieu des marais.

C'est alors que j'ai découvert le livre d'André Bonnard La Civilisation grecque, où il y avait une photographie d'oiseau (j'ai cru y voir un héron) au milieu de pierres, dans les ruines d'un ancien palais. Plus tard, en retrouvant le livre, je me suis aperçu que les oiseaux $\left(\right.$ des grues $\left.^{5}\right)$ et les ruines de l'ancienne Persépolis étaient 
SLOVO

264 À l'Est de Pixar : le film d'animation russe et soviétique - no 48/49

deux photos différentes. Mon imagination les avait réunies et en avait fait une seule image.

Avec des corbeaux, le problème aurait été différent, mais un héron et une cigogne sont de nobles oiseaux, blancs et beaux. C'est cela qui donnait sens à mon film, le reste était de la technique.

Mais, à son tour, l'espace a dicté les conditions de l'action. D’emblée il y a eu un feu d'artifice, une balançoire pour le héron, un banc de fonte cassé. Tous ces détails s'imposaient d'eux-mêmes et, avec le dessinateur, nous ne faisions qu'adapter l'histoire à l'espace que nous avions choisi.

Le conflit entre le héron et la cigogne a immédiatement pris la tournure d'une comédie triste à la Tchékhov, avec des héros qui sentent qu'il en faut si peu, qu'il suffit d'attendre seulement un tout petit peu - et le bonheur sera là.

\section{L'air est la matière du plan (I, p. 152)}

Je vais évoquer maintenant le ciel dans Le Héron et la Cigogne. Il n'a pas été dessiné, il a été formé par la lumière.

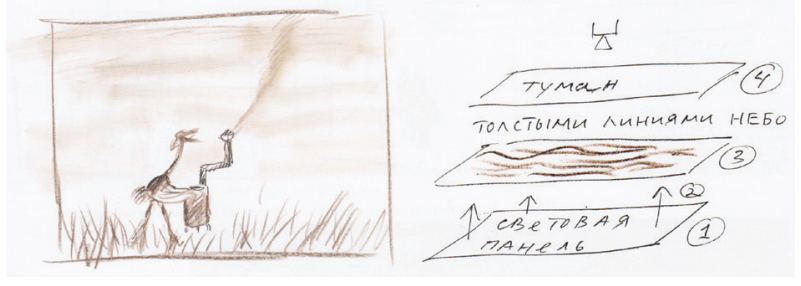

Figure 8

Youri Norstein : dessin de pour Le Héron et la Cigogne et schéma de la prise de vue

Voici comment : il y a un panneau lumineux (1) d'où provient la lumière (2). Ensuite, il y a encore un autre niveau (3) où il y a du cellulö̈d sur lequel sont dessinées des rayures ondulées (4). Au niveau supérieur (5), il y a une surface mate les futurs nuages. En élevant la surface mate au-dessus des rayures ondulées, nous avons adoucis ces dernières jusqu'à ce qu'elles deviennent des nuages ondulés. Nous avons ajouté sur la surface mate une couche double, triple de celluloïd poussiéreux pour épaissir le ciel. Ainsi, nous avons pu réguler la densité de l'image des cigognes.

oblige le traducteur à ruser, c'est ainsi que l'on traduit par cigogne. Une autre difficulté de traduction est qu'en russe le héron est au féminin et la grue au masculin, ce qui inverse les genres. Le caractère schématisé des dessins du film a permis ce tour de passe-passe lexical (n.d.t.). 
Le petit hérisson (I, p. 159-160)

Je ne sais quel instinct ou quelle raison ont arrêté mon choix sur ce conte. Quelques vagues associations m'étaient venues à l'esprit à sa lecture, qui n'avaient rien à voir avec le texte. Je pressentais un certain village, quelque part. Du brouillard. Un troupeau sur la route, qui se confond avec la chaussée. On ne voit pas les pattes des vaches, seulement leurs dos, qui s'écoulent dans le village comme un fleuve. (Vous vous souvenez de mon exemple du début sur les métaphores dans la poésie tamoul : l'auteur décrit une route en forêt et la compare avec le dos d'un éléphant qui court.) Et soudain j'entendis une vache meugler au village. Juste ça ! Juste un bruit, c'est tout. Seulement, il n'y avait là aucun lien avec le hérisson, pas le moindre lien avec le conte.

À présent, je pense que c'était une question d'espace. J'ai tout simplement ressenti ce conte de façon spatiale, comme un écho. J'ai senti que là-bas, quelque part dans le lointain, derrière la toile de l'écran, il y avait une sphère qui reflétait le son et le renvoyait ici. Tout cela devenait une image.

C'est vraiment difficile d'expliquer combien l'imaginaire est fantasque, pourquoi une image et non une autre.

Il se peut que le puits sans fond et le son qu'il renvoie soient sortis de cette sensation. Et l'obscurité. Lorsque nous dessinions le puits, j'écrivis très en détail cette scène. Je précisai l'odeur des poutres, recouvertes d'une moisissure veloutée, le léger chuchotement de la terre qui s'échappait de dessous les poutres. On ne retrouve pas ces détails dans le film, mais ce fut son premier écho.

Je proposai ma version personnelle du scénario. Il est très simple. Un petit hérisson rend visite chaque soir à son ami Ourson. Mais un jour, il se perd dans le brouillard et a la vision d'une autre vie, inconnue de lui. Il se heurte à un mystère. Il erre dans le brouillard et entend l'appel d'Ourson. Un détail important : ce cri élargit l'espace. Cela me fait penser à la fin du récit de Tourgueniev Les Chanteurs, lorsque, dans le champ envahi d'obscurité, un cri d'enfant retentit : « Antro-o-oopka, Antro-o-o-opka! ». Quand Hérisson retrouve enfin son ami Ourson, il n'est plus le même, il sait quelque chose que ne sait pas son naif ami (fig. 9).

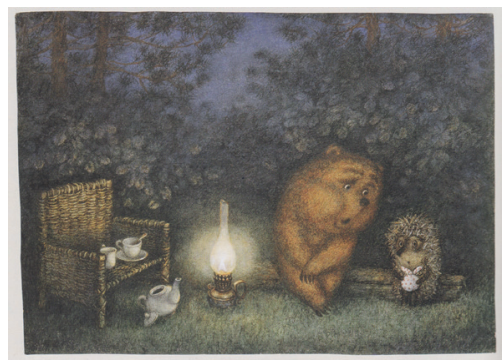

Figure 9

Ourson et Hérisson, Le Petit Hérisson dans le brouillard 
SLOVO

266 À l'Est de Pixar : le film d'animation russe et soviétique - no 48/49

Il me semblait que le film devait être construit solidement, comme on monte et renforce les murs d'une maison. Au début, c'était plus une étude impressionniste qu'un scénario. De manière générale, pour moi, le scénario n'est pas la base d'un film, ce serait plutôt la mémoire qui s'enflamme, qui dépasse les limites d'une vie. Un plan est une éruption de conscience.

Plusieurs secondes d'action en un instant.

Une condensation colossale d'énergie créatrice.

Des fragments épars d'une fresque qui s'effrite et qu'il faut restaurer.

Puis, quand on a sous les yeux un parchemin et son texte, alors seulement en sortent peu à peu des liens visuels. C'est une sensation purement physique. On voit le papier se transformer en dessins, en fioritures et se couvrir d'appogiatures. Les images sont floues, c'est pourquoi je préfère le vocabulaire musical. Comme si tout était déja là, à l'intérieur, et que soudain une cellule vivante en jaillit.

Je vois le papier se matérialiser en objets qui s'élèvent au-dessus de lui, telle la vapeur au-dessus de l'eau bouillante.

Le film ne peut se développer par lui-même quand une association personnelle et vivante pénètre dans sa structure. Le texte n'est plus qu'une proposition subordonnée.

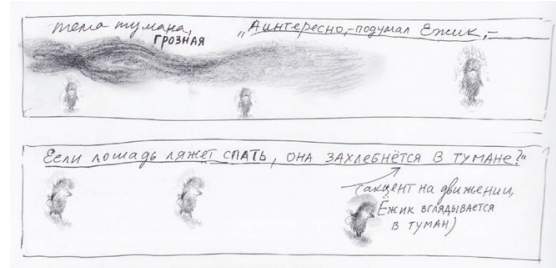

Figures 10 et 11

Représentation de la musique dans $L e$ Petit Hérisson dans le brouillard

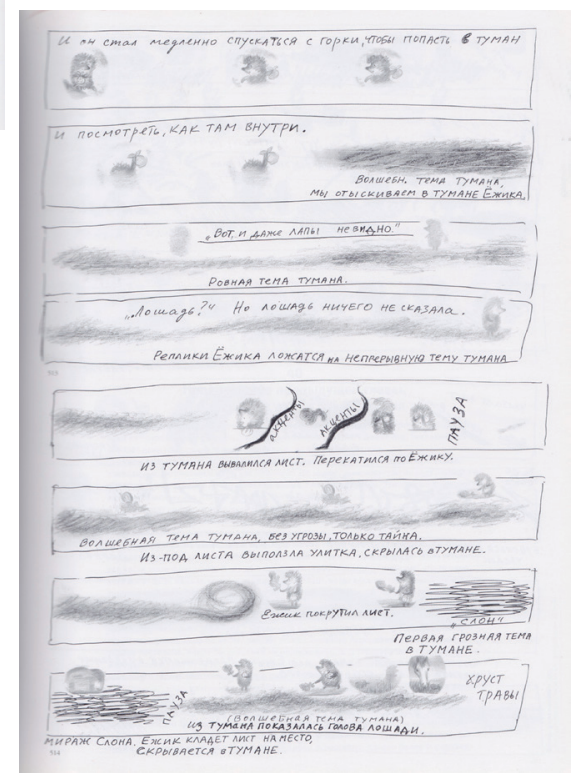


Si l'on dresse une courbe (I, p. 180)

Si l'on dresse une courbe de ce long extrait (trois minutes au moins), on voit qu'elle présente des alternances de rythmes et de pauses.

Progressivement, les nouveaux rythmes se font de plus en plus fréquents et hachés, jusqu'à ce que cet énorme flot rythmique prenne une allure de folie, d'un mouvement ininterrompu et chaotique. C'est à peu près le graphique du dessin et du son. Je dois dire que j'ai été influencé par la lecture de l'article d'Eisenstein « la montage vertical ». Ce texte me paraît encore plus adapté au film d'animation qu'au cinéma en prises de vue réelles.

Le Conte des contes (I, p. 243-244)

La chaleur du soleil de l'été de la Saint-Martin. Des tables, des tabourets, sortis de l'appartement communautaire... Les voisins assis à table avant de quitter cette maison pour toujours...

Il vivait en moi, ce film, bien longtemps avant que je ne songe à devenir réalisateur.

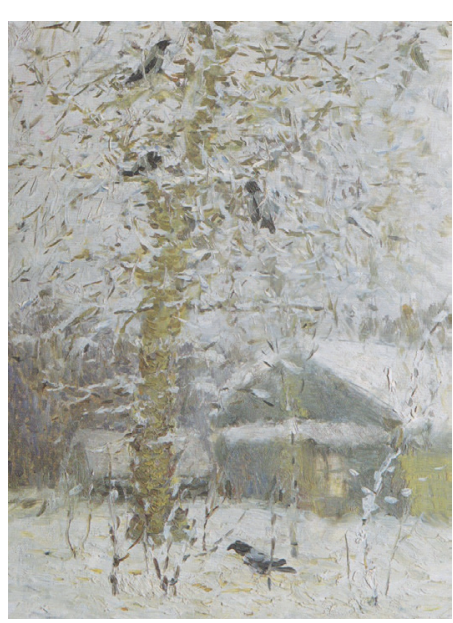

J'ai un croquis dont je ne me rappelle pas la date, à la fin de mes études d'arts plastiques ou peu après, à mon arrivée dans les studios, une étude de corbeaux sur un arbre enneigé (fig. 12), c'est Le Conte des contes.

Et un autre dessin assez enfantin: la mère Varia, Varvara Nikititchna Timokhina, notre voisine du quartier de Marina Rochtcha (fig. 13), c'est aussi Le Conte des contes.

Figures 12 et 13

Youri Norstein : Corbeaux sur un arbre enneigé et Vieille au coin du feu (Varvara Nikititchna Timokhina), études, 1955

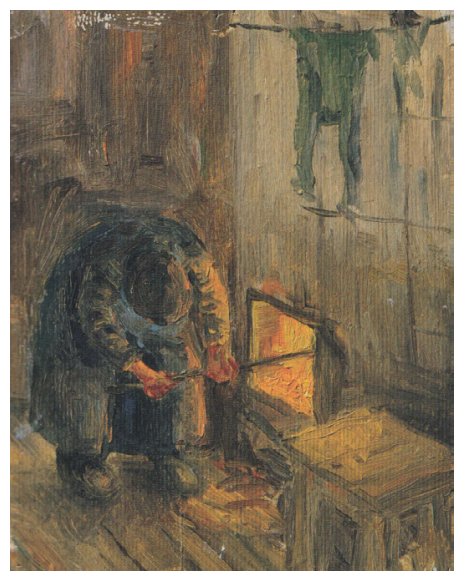


SLOVO

268 À l'Est de Pixar : le film d'animation russe et soviétique - no 48/49

L'origine n'est pas là. Ni dans la berceuse que, par la suite, je chantais sans cesse, j'ignore pourquoi. Et quand et pourquoi le loup est apparu, je ne le sais pas non plus. Bien que ce soit un personnage de l'enfance. Ce personnage... ce héros a habité mon enfance. Je pense qu'il a continué d'habiter ma maison quand moi, je l'ai quittée, et je ne sais ce qu'il est devenu lorsqu'on l'a démolie. Chaque maison, on le sait, a et doit avoir son « esprit ».

Mais d'abord, il y avait la nappe, blanche, grande, assez pour recouvrir les tables, dehors dans la cour. Des tables apportées des logements communautaires. Le soleil était de la partie, il faisait chaud, c'était l'automne, l'été de la Saint-Martin. Les voisins se serraient autour des tables avant de quitter leurs « pénates natales » pour toujours.

Plus tard, on m'a accusé d'avoir emprunté la nappe à Tarkovski, jamais de la vie !

Un nourrisson au sein, et quel sein !

Un souvenir de 1945, ma chère tata Bella...

Elle travaillait dans l'armée de l'air où elle avait trouvé un mari. Elle est rentrée $\mathrm{du}$ front en 1945, enceinte, «ventrue » comme aurait dit Pouchkine, elle a accouché, et le bébé est mort deux semaines plus tard d'une septicémie...

J'avais à peine quatre ans mais je m'en souviens très bien. Je me souviens qu'elle regorgeait de lait, et le matin, elle le tirait dans une cruche brune qui nous est restée longtemps.

Je me réveille, la maison est plongée dans une obscurité couleur de cendres, sa poitrine blanche émerge de sa chemise ouverte, j'entends le son du jet dans la cruche. Je me rappelle le goût de ce lait, sa lumière, sa chaleur. Le côté obscur des

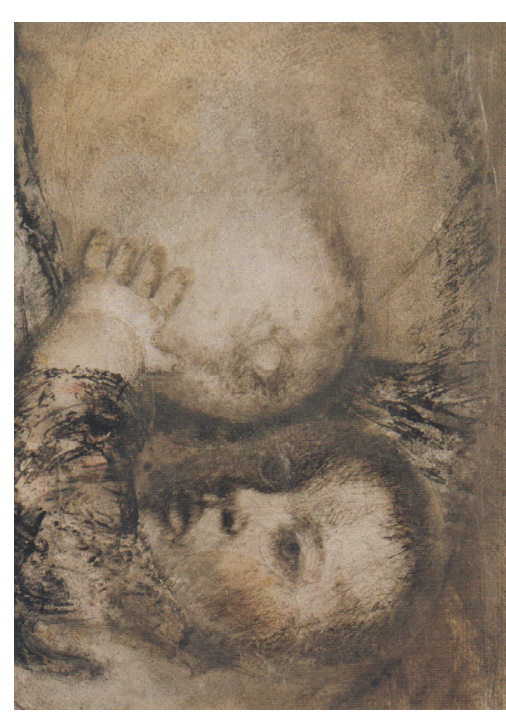
souvenirs liés à ces images vit en moi. Mon compteur Geiger se met à vibrer lorsqu'il approche ces souvenirs.

La séquence où le bébé tète au sein émane de diverses impressions.

J'ai parlé de ma tante, mais j'ai aussi une photo sur laquelle ma Frantcheska est assise au bord du lit en chemise de nuit et tient dans ses bras notre fils Boris à peine réveillé.

J'ai demandé à Frantcheska de dessiner sa tête pratiquement grandeur nature pour le plan. En outre, nous avons bien observé comment est faite la poitrine de « La nuit », de Michel-Ange sur le tombeau des Médicis. 


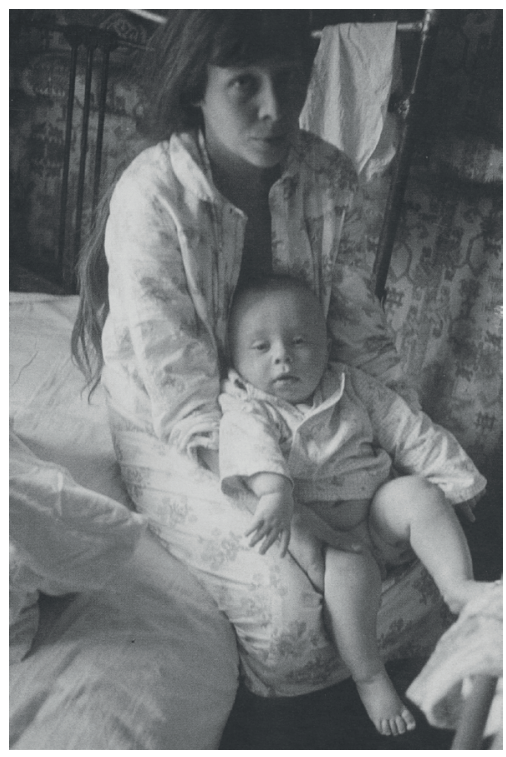

Figure 14 et 15 Youri Norstein, Frantcheska donne le sein à Boria et Frantcheska et Boria, 1968

Le bruit de succion du bébé au sein nous a été fourni par hasard grâce à la chienne d'une amie qui venait de mettre bas ses chiots. Un petit dernier traînait au sein et nous avons enregistré son bruit de succion, qui nous a servi de bande son pour cette séquence.

\section{Le Conte des contes est donc aussi un film sur l'enfance (I, p. 254-255)}

Le film devait s'appeler Le Loup viendra, et je pense encore maintenant que ce titre était plus juste. Malheureusement, on l'a changé après la première projection, sous un prétexte que j'ai du mal à comprendre, il sous-entendrait une menace. Je ne vois pas bien. Nous n'avons pas inventé non plus le titre Le Conte des contes. C'est le titre d'un poème de Nazym Khikmet que j'aime beaucoup, comme j'aime aussi les poèmes de Pablo Neruda, par exemple « Une nappe pour tous ». Tous ces poèmes, qui ont toujours vécu et continuent de vivre en moi, ont pris leur place dans le film sans qu'on les oblige à venir. Tout le film est fait de ce qui me fait vivre, de ce qui me plaît, de ce que je n'accepte pas, de ce que j'aime, de ce que j'ai assimilé, de ce que j'ai surmonté, de ce que j'ai compris.

Pourquoi y avoir introduit le thème de la guerre ? J'ai connu des femmes, des sœurs, des parents de soldats qui n'en sont pas revenus. J'ai connu bien des gens qui recevaient les annonces des morts. Mais ce n'est pas seulement pour cela. Le thème est venu tout seul. Oui, une guerre, c'est une guerre contre un ennemi, mais ce n'est pas assez pour défendre sa patrie. Chaque combattant avait quelque chose de personnel, une individualité sans laquelle il ne pouvait y avoir de social. Chacun avait sa maison, qu'il défendait, chacun avait ses parents et ses proches. La guerre était animée par ce mouvement général. Là, nul n’était besoin de mentir, ni d'attendre des visiteurs nocturnes dans leurs $\ll$ maroussias $^{6} \gg$ noires.

6. Voitures de police cellulaires (n.d.t.). 
SLOVO

270 À l'Est de Pixar : le film d'animation russe et soviétique - no 48/49

Le thème de la paix est venu équilibrer celui de la guerre, pas la paix tout court, mais une paix éternelle, l'Éternité... Voici déjà plus de soixante ans que cette guerre est finie et nous ne savons toujours pas ce que signifie vivre en paix. Faire en sorte que les générations nouvelles puissent vivre en paix, sans penser à la guerre ou à une mort violente, pour qu'elles puissent réfléchir à ce qu'est la vie, au sens que l'on peut lui donner. Le sens que nous donnerons à notre vie, c'est l'avenir que nous nous construirons. Notre espace spirituel s'élargira ou se rétractera en fonction de notre soif de sens.

D'une part ça, et d'autre part le motif de la vie éternelle. Non pas sans doute le repos dont parle Woland ${ }^{7}$. Pour moi, c'est une sensation de permanence éternelle de la vie. Nous pouvons tous nous rappeler des moments où nous avons ressenti une harmonie parfaite avec le monde. De tels moments sont rares, et ils ne peuvent durer, mais ils nous donnent la force de vivre. Ils sont propres à chacun et peuvent paraître surprenants ou étranges à un autre. Des moments simples sans lesquels on ne pourrait vivre.

Une invitation à partager un repas...

Un compagnon de route...

Un secret d'enfant...

La feuille blanche sur la table du poète...

Un arbre sur terre...

Ce doit être un film sur la mémoire.

Vous vous souvenez combien les jours étaient longs dans votre enfance?

Chaque jour se suffisait, l'aujourd' hui s' accomplissait aujourd'hui, et le bonheur de demain serait à voir le lendemain.

Les vérités étaient simples, tout nouvel objet vous plongeait dans l'émerveillement; l'amitié et la camaraderie, c'était le plus important.

Cette manie d'éternellement repousser la vie au lendemain, beaucoup l'acquièrent avec l'âge. On ne sait plus comment on vit, l'amitié, ce n'est plus ça, les joies que procurent le soleil, la neige, le vent, une promenade, une assiette toute propre, des chiens, des chats, ne sont plus perçues comme des joies (fig. 16).

Soyons épargnés de vivre ainsi dans l'attente de notre destin. Mais le film ne traite pas de cela.

7.Le personnage du diable dans Le Maître et Marguerite de Mixail Bulgakov (n.d.t.). 


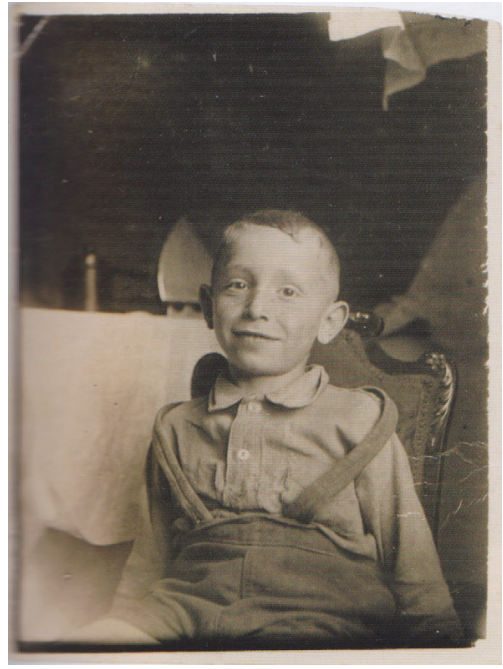

Figure 16

J'ai cinq ans. Je viens de rentrer. L'oncle Iossif a réglé son appareil photo, il m'a assis sur une chaise près de la fenêtre, n'a pas remonté ma bretelle, ne m'a pas peigné, il m'a pris en photo tel que j'étais : avec ma chemise qui dépasse de mon pantalon et mon col retourné.

Ce film doit avoir un poète dans le rôle principal, un poète qui n'apparaîtra d'ailleurs pas forcément à l'écran, on pourra peut-être y voir ses vers, par exemple « Le Conte des contes $\gg$ de Nazym Khikmet dans la traduction de Mouza Pavlova :

Nous sommes là au bord de l'eau,

Le soleil, le chat, le platane, moi et notre destin.

L'eau coule fraîche

L'arbre est très haut,

J'écris des vers,

Le chat somnole,

Le soleil chauffe.

Dieu soit loué, nous vivons !

L'éclat de l'eau nous frappe au visage,

Nous tous, le soleil, le chat, le platane, moi et notre destin.

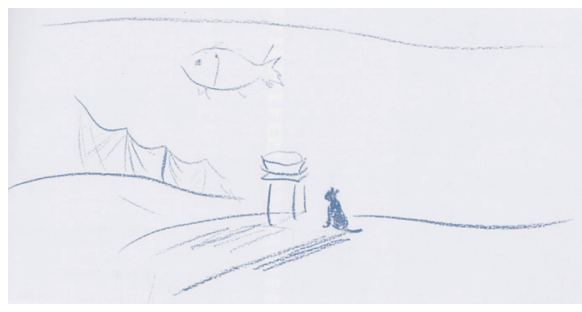

Figure 17

Le Chat et le Poisson

Youri Norstein, 2003
Mais il faut un chat à l'écran, un être plein d'amour et de fidélité (fig. 17), et une chaussure, une seule, trouvée par des enfants dans les ordures - qui a bien pu la poser là, neuve, avec sa semelle intacte? Une souche de bouleau qui, au printemps, comme chez Tvardovski, «bouillonne d'une écume rose ». Plein de papillons, des hannetons, 
SLOVO

272 À l'Est de Pixar : le film d'animation russe et soviétique - no 48/49

des abeilles amaigries par l'hiver volent pour se rassembler au festin. La pluie arrose la terre, remplit la chaussure, la souche, lave les pavés de la route et au bout de la rue, un crépuscule du soir qui se lève et va durer longtemps.

Du linge sèche sur des fils, un taureau avec un anneau dans les naseaux, animé de passions sanglantes, terribles. Un gars sur sa béquille et sa jambe unique, notre voisin revenu de la guerre... Notre voisin qui n'a qu'une seule chaussure...

Le sujet peut être organisé très simplement, mais il faut qu'il soit un sujet bien particulier, un sujet-accordéon qui se plie et se déplie et qui se résume à la fin en un seul mot : nous vivons. Parce que nous avons vécu notre enfance à la fin de la guerre et devons toujours nous souvenir que le bonheur, c'est chaque jour de paix. Chaque jour.

\section{Chaque jour de tournage est différent des autres. Et on doit tout le temps percevoir cette différence.}

Imaginons la création d'un film comme la composition d'un poème, certains s'écrieront : « Ce n'est pas rentable », et ils auront raison. Le cinéma, c'est vrai, est un plaisir qui revient cher. Mais l'animation est une exception. Pas dans tous les cas, mais parfois, c'est une exception.

Il peut arriver que le scénario s'échappe, comme deux trains roulant sur deux voies parallèles qui s'écartent l'un de l'autre. Pour La Renarde et le Lièvre, le tournage correspondait très précisément au scénario, jusqu'aux détails du jeu des personnages. Pour Le Héron et la Cigogne, la concordance était parfaite au plan près. Dans Le Hérisson, il y a eu des choses qui ont disparu et d'autres qui sont apparues. Quant au scénario du Conte des contes, il a beaucoup évolué au fur et à mesure des prises de vue. On tournait comme si les séquences étaient une réaction immédiate à ce qui se passait dans le film. Chaque jour de tournage était différent des autres, et il fallait percevoir en quoi. L'art est art parce qu'il sélectionne. Sinon ce serait seulement la vie réelle.

Y aurait-il une nécessité de l'art si, dans la vie réelle, nous pouvions nous ouvrir directement à l'autre, dans son unicité, comme nous nous ouvrons dans les moments de création ? Peut-être faut-il chercher des réponses auprès des tribus qui vivent encore selon les lois de la nature.

Le substitut de l'art est le rituel, qui est à la fois étude, loi, religion et philosophie. Caparaçonnés dans nos villes, éparpillés, sans aucune force qui nous relie les uns aux autres (la religion peut-être ?), nous cherchons des voies qui nous mèneraient les uns vers les autres. L'art est l'une de ces voies. Ses métaphores ouvrent les portes du monde. L'art rend visible le caché, il transfigure le réel par des paroles inattendues. L'art enflamme la réalité, nous fait voir l'invisible, il nous imprègne de sympathie, de honte, de conscience morale. L'art est le médiateur de tout ce qui vit. Il rétablit un ordre harmonieux. 


\section{Le Manteau}

J'aurais du mal à expliquer ce que j'ai voulu exprimer dans ce film (II, p. 11) Il y a bien quelque chose qui a donné naissance au film, mais c'est très ténu. Je faisais des dessins pour Le Conte des contes, je pensais au film, et j'ai fait un dessin - il m'est bien sûr plus facile de traduire mes pensées en dessins qu'en mots : sur mon dessin, un homme était assis sur un lit dans l'obscurité du soir. C'est tout, rien de plus ; et j'ai eu une impression de déjà-vu. Le Manteau vient de là, de ce sentiment, ou peut-être d'autre chose, de quelque chose de l'enfance, je ne sais pas.

Les expériences de vie dans l'enfance sont plus fortes qu'à l'âge adulte. Le monde fait face à l'enfant, il ne peut passer ni à droite, ni à gauche. Avec le temps, nous saisissons nos impressions de biais, nous les évitons, nous avons d'autres buts, la plupart du temps absurdes. Gogol nous raconte l'histoire d'un homme qui est adulte, mais qui a la psychologie d'un enfant. Quand j'étais moi-même enfant, j'étais gêné par ce décalage, mais je n'en n'étais pas conscient. C'était quelque chose qui m'avait visiblement marqué, mais que je n'aurais pu expliquer à personne, avec mon raisonnement de douze ans d'âge et des sentiments tellement plus forts que le raisonnement ; il est impossible de les expliquer, c'est pourquoi ils l'emportent. C'est aussi pourquoi j'aurais du mal à formuler clairement ce que j'ai voulu exprimer dans ce film. Sûrement montrer le sentiment de la honte, que nous éprouvons tous les uns devant les autres ; chacun, au fond de soi, a honte de quelque chose et voudrait en parler, ou bien on a honte pour autrui.

Le Manteau, je pense, provoque un sentiment de honte.

Honte de ce qu'un homme ne veut pas comprendre ce qu'éprouve, ce que vit l'autre. Et si soudain il le comprend, il a honte de sa propre vie. Le sentiment exagéré de sa propre dignité, de confiance en soi, de supériorité, n'élève pas l'homme mais le pulvérise. En poussière, en poudre. Son corps continue d'exister, de parler, de manger, de jouir de sa puissance. Mais quand soudain on a honte de la fausseté de sa vie - de s'être trompé, d'avoir un jour offensé quelqu'un d'inférieur, de moins important - alors on grandit en tant que personne. Il ne s'agit pas tant du fait qu'on a blessé quelqu'un. Des péchés, nous en commettons tous, nous devons simplement en garder le souvenir. Et nous en repentir.

Oh, comme nous avons exagéré notre compassion envers le petit homme, et comme notre orgueil est flatté par la démonstration de nos nobles élans (II, p. 12)

Le Manteau est imprégné de ce sentiment de honte, car, en fin de compte, le fantôme d'Akaki Akakievitch se venge, et il ne se venge pas seulement d'une seule personne, mais il se venge du monde entier. Il arrache les manteaux de tout le monde, y compris d'un petit homme qui ne peut se protéger, « un flûtiste qui avait 
SLOVO

274 À l'Est de Pixar : le film d'animation russe et soviétique - no 48/49

fait jadis partie d'un orchestre ». Cette nouvelle est vraiment terrible.

Le Manteau est le fonds génétique de la honte humaine. Imaginons un instant une société idéale, elle ne pourrait exister sans Le Manteau, sinon nous n'aurions plus de reflexe d'auto-défense.

Il fut un temps où l'on parlait du thème du « petit homme » dans Le Manteau. On se trompait. Oui, bien sûr, Akaki Akakievitch est un «petit homme », dans la mesure où il occupe un poste modeste, ne dirige rien, n'a pas de mission particulière sur terre. Il ne fait qu'accomplir son petit travail tranquille.

Mais pourquoi parler de « petit homme » alors qu'au fond, c'est un personnage cosmique qui projette sur lui tout le monde environnant. Tout son psychisme, sa nature d'artiste s'expriment dans la lettre qu'il est en train de tracer. Cette participation au monde fait de lui une personne exceptionnelle et une icône unique.

Les critiques littéraires ont coutume d'écrire d'une adaptation qu'elle est « une fine lecture de l'œuvre littéraire ». Il n'y a pas de pire injure pour un film. Transposer à l'écran les nouvelles de Gogol ne pose pas de problème. C'est le passage d'une technique à une autre. Le problème est ailleurs. La fidélité d'un film à une œuvre littéraire veut dire que le réalisateur est taraudé par les mêmes problèmes que l'auteur du livre, avec la même force. Sinon, c'est du temps de travail qui donne un film banal dont on dira ceci ou cela dans une biographie. Et si le film est raté, on dira: «Bon, il reste Gogol». Facile aussi. Mais on ne pose pas la question: pourquoi Gogol, pourquoi Le Manteau? Cette nouvelle est immortelle non pas seulement en tant qu'œuvre de Gogol; elle est immortelle parce que la vie est immortelle dans toute son horreur. Cette nouvelle doit avoir sa place même dans un monde où la vie serait bienheureuse et enchanteresse. De même que l'enfer existe dans la conscience du croyant, il ne l'a pas vu, mais il sait que les péchés seront expiés. Ainsi, le Manteau contient une exhortation, un code, qui doit rester pour toujours une réserve génétique de prévention pour l'humanité.

Quand un artiste travaille sur un sujet religieux, il crée ses propres illustrations du Livre Saint. Le texte biblique est un plasma moral d'où s'écoulent des flots salutaires qui nous éclairent depuis plus de deux mille ans. Il y la même source infinie d'énergie dans l'Ancien et le Nouveau Testaments, qu'on ne peut même pas comparer avec la fission de l'atome.

Le schéma des prises de vue (II, p. 126)

- Comment est construite la lumière dans l'épisode où Akaki Akakievitch est dans sa pièce, à son bureau, et quel est le schéma de la prise de vues?

— Voici comment est la mise en scène : le bureau, le lit, le coin, là, Akaki Akakievitch est assis, là une bougie est allumée, devant lui il 
y a un pupitre-miroir, il pose une feuille dessus, l'ombre est derrière lui (fig. 18).

Donc, le fond est éclairé, et la bougie est éclairée (3), une lumière générale douce sur

Figure 18 -Akaki Akakievitch à son bureau, dessin de la lumière et Figure 19 - Le dessin de la lumière, schémas

Youri Norstein, 2003

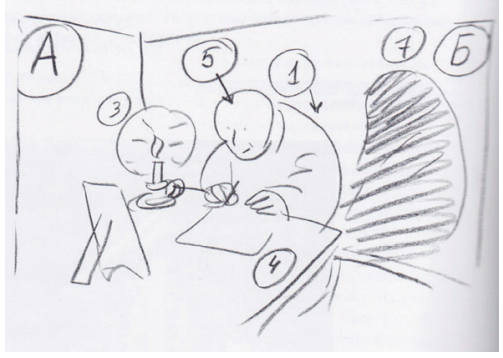

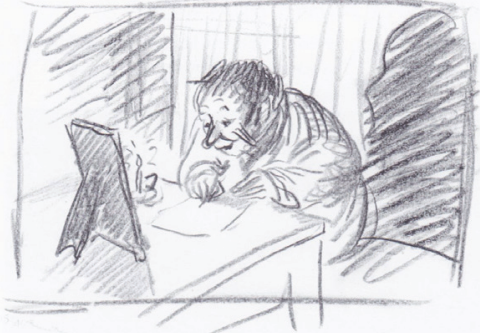

tout le décor et l'ombre (7), qui, bien sûr, se déplace en même temps que lui. Là il y a le lit, là le mur - il est au second plan, nous désignerons la partie gauche par A, la droite par B.

La source d'éclairage (6) éclaire Akaki Akakievitch, l'ombre tombe sur le deuxième niveau, où se trouve l'ombre d'Akaki Akakievitch (7). De plus, sur la vitre supérieure, hors cadre (8), il y a des petits morceaux de papier noir, avec lesquels nous pouvons faire l'ombre sur le visage d'Akaki Akakievitch (5), en couvrant ou diminuant la lumière hors cadre. Nous pouvons changer la configuration de la lumière, en atténuant la silhouette (1). La bougie est éclairée par une lumière propre (1). Qui vient d'une source d'éclairage très étroite (9). Sur la vitre d'en haut il y a un filtre, un diffuseur (10). $\mathrm{Du}$ grain photographique très très fin est imprimé sur le celluloïd. C'est, bien sûr, le travail de l'opérateur, et ici, ce qui est très important c'est comment il se représente le plan. Mais la lumière, nous l'établissons

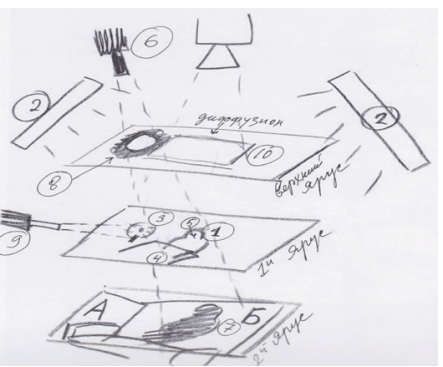

Figure 20

Schéma du dispositif d'éclairage Youri Norstein, 2005 ensemble. La distribution de la lumière n'est pas seulement liée au travail de l'opérateur ou de l'exposition, elle est aussi liée au jeu du personnage. Le travail avec la lumière 
SLOVO

276 À l'Est de Pixar : le film d'animation russe et soviétique - no 48/49

est très complexe.

La lumière, c'est la matière du plan.

La lumière, c'est le personnage, et nous jouons avec le flux de lumière.

Le faisceau de lumière, nous devons l'amener, le « lier » à Akaki de manière à ce que quand nous bougeons le personnage, nous bougons aussi la lumière, comme si elle était enchaînée à lui.

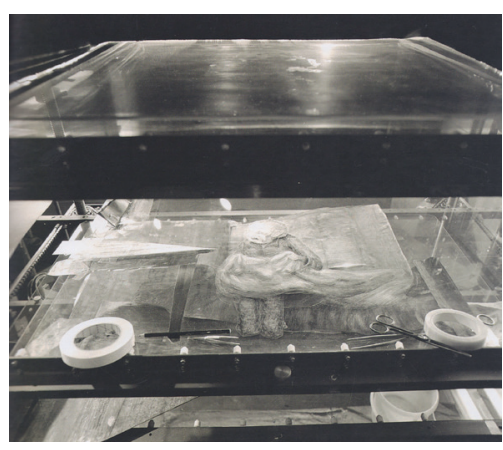

Figure 21

La silhouette d'Akaki Akakievitch sur les vitres sous la caméra (c) Igor Skidan-Bossinela, 1995

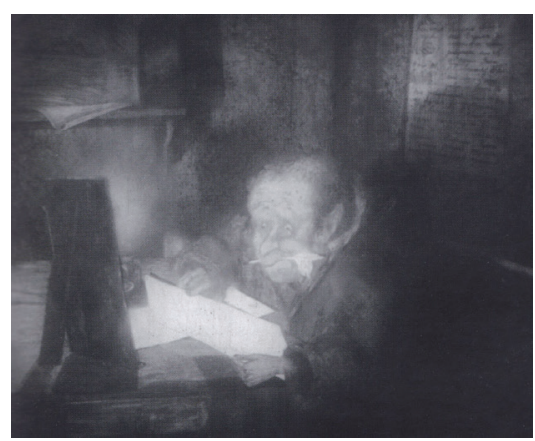

Figure 22

Akaki Akakievitch écrit, scène du Manteau, opérateur Aleksandr Joukovski, 1985

Résumé : les extraits choisis de Neige sur herbe [Sneg na trave] de Youri Norstein décrivent le processus de création artistique du cinéaste à travers ses réflexions théoriques et ses explications sur ses influences picturales, narratives et autobiographiques. Ils permettent de découvrir ses dispositifs de tournage et de mieux comprendre l'œuvre de ce cinéaste incontournable de l'animation russe et soviétique.

Mots-clefs : Youri Norstein, animation, laboratoire du créateur, autobiographie, réflexion théorique.

Abstract: the excerpts translated from Yuri Norstein's Snow on Grass [Sneg and trave] describe the artistic process of the filmmaker through his theories and through his influences (paintings, narratives, autobiography). They disclose the concrete ways in which he stages and films. The reader thus gains a deeper understanding of Norstein's oeuvre, which plays an essential part in Soviet and Russian animation. 
Keywords: YuriNorstein, animation, creator's laboratory, autobiography, theoratical reflexion.

Абстракт: представленныц в этом издании отрььвки из Снега на Траве Юрия Норитейна описььвают творческий процесс режиссёра, а также дают представление о его теории анимации и о повлиявиих на него художественных произведениях (живописи, митературь) и эпизодах из его жизни. Они дают конкретную информачию отом, какНоритейн снимает свои фильмь. Читатель сможет таким образом глубже понять творчество Юрия Норитейна, одного из ведуших мастеров российской и советской мультипликаиии.

Ключеввге слова: Юрий Норитейн, анимачия, лаборатория художника, автобиография, теоретические размышиления. 